\title{
Ergonomic Assessment of an Active Orthosis for the Rehabilitation of Flexion and Extension of Wrist
}

\author{
Adriano O. Andrade ${ }^{1[0000-0002-5689-6606]}$, Julien Bourget ${ }^{1,2[0000-0003-0054-2777]}$, Samila \\ Costa $^{1[0000-0001-7875-4206]}$, Adriano Pereira ${ }^{1[0000-0002-1522-9989]}$, Michael I. Okereke ${ }^{3[0000-0002-}$ \\ 2104-012X] and Marcus Fraga Vieira ${ }^{4[0000-0001-9096-1603]}$ \\ ${ }^{1}$ Centre for Innovation and Technology Assessment in Health, Federal University of Uber- \\ lândia, Uberlândia, Brazil. \\ ${ }^{2}$ Biomedical Engineering, University Claude Bernard Lyon 1, Polytech Lyon, Lyon, France. \\ ${ }^{3}$ Department of Engineering Science, University of Greenwich, London, United Kingdom. \\ ${ }^{4}$ Bioengineering and Biomechanics Laboratory, Federal University of Goiás, Goiânia, Brazil. \\ adriano@ufu.br, julien.bourget@etu.univ-lyon1.fr, \\ samilac.costa@gmail.com, a.alves.pereira@uol.com.br, \\ M.I.Okereke@greenwich.ac.uk, marcus.fraga.vieira@gmail.com
}

\begin{abstract}
Muscular stiffness and limb rigidity are two main consequences of Parkinson's disease. These motor symptoms may be present in distinct parts of the body, influencing functional tasks executed by hands. To aid people suffering from these motor symptoms, we developed an active wrist orthosis whose purpose is to enable increase the flexion and extension range of motion of the wrist joint. We identified five relevant ergonomic variables that should be considered when using the orthosis in the clinical practice: (i) device stability, (ii) forearm position; (iii) muscular strength; (iv) amplitude of motion; and (v) mass of the device. These variables were identified based on the observation of movements while users executed the flexion and extension of the wrist with and without the device. In this research, we present a description of the developed orthosis and an evaluation of the ergonomic variables (i), (ii) and (iii). An enhanced support structure has been used with the orthosis and shown to lead to a stability improvement. Electromyographic analysis showed that the use of the orthosis does not introduce undue muscular load on the user at distinct angular positions of the forearm.
\end{abstract}

Keywords: Parkinson's Disease, Orthosis, Ergonomics, Electromyography.

\section{Introduction}

Parkinson's disease (PD) is a neurodegenerative disease that leads to progressive loss of dopaminergic neurons from the substantia nigra [2]. Dopamine deficiency produces a severe extrapyramidal system effect, resulting in decreased muscular strength [5]. These pathophysiological changes generate typical neurological symptoms found in patients with PD such as bradykinesia, rest and postural tremor, postural instability, and muscular stiffness [3].

A research area that can contribute to patients with PD is the provision of physiotherapeutic aid based on robotic devices for safe and intensive rehabilitation of 
individuals with light to severe motor deficits. Such use of robotic devices has been shown to partially restore the function of the upper limb of individuals affected by PD $[4,7]$. The work of Sangha, et al. [6] is an example of the rehabilitation of flexion and extension movements of the wrist. However, as in most of the studies in this area, the authors do not present ergonomic assessment of the developed device. The ergonomics of any orthosis is vital for eventual adoption in PD-related physiotherapy. In this study, we present the description of an active wrist orthosis and the evaluation of ergonomic variables that may affect its practical use.

\section{General Description of the Orthosis}

The active wrist orthosis (Fig. 1), was developed in the Centre for Innovation and Technology Assessment in Health (NIATS) of the Federal University of Uberlândia. The current design has been patented (INPI-BR10 2014023282 6). Ethical approval for the development and assessment of the orthosis was granted by the National Committee for Ethics in Research (CAAE: 07075413.6.0000.5152).

The orthosis was manufactured mainly from aluminum and its overall weight is 909 g. The actuator is a linear DC-Servomotor (LM 2070-080-11, Faulhaber, Germany). Programmed motion of the orthosis is achieved via an external motion controller (MCLM-3006-S-RS, Faulhaber, Germany), which is not shown in Fig. 1. As the focus of this research is the ergonomic assessment of the orthosis in a passive scenario (i.e., without considering linear forces exerted by the actuator), all the evaluations were performed without the influence of the actuator, which means that it was turned off.

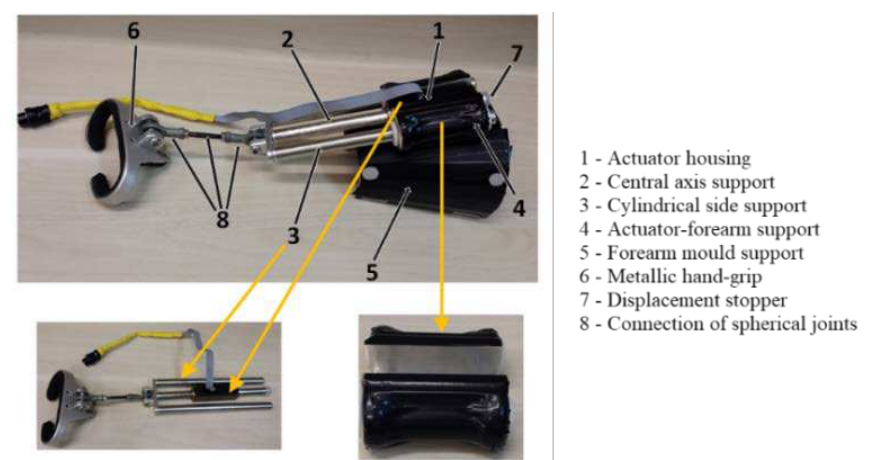

Fig. 1: Identification of the parts of the orthosis.

\section{Methodology for Ergonomic Assessment}

Based on preliminary tests with the orthosis, five ergonomic variables were identified: (i) device stability, (ii) forearm position; (iii) muscular strength; (iv) amplitude of motion; and (v) mass of the device. In this paper it is presented only results concerning the analysis of the variables (i), (ii) and (iii). It is expected that results related to variables (iv) and (v) are presented in complementary future work. 


\subsection{Orthosis Stability}

For effective transfer of the actuator force to the hand, the orthosis must be securely fixed on the forearm. A special under-forearm support was manufactured (designed and 3D-printed) to securely fix the orthosis.

To evaluate the gain in stability (i.e., the steadiness of the orthosis on the forearm) provided by the support, a volunteer carried out the flexion and extension of the wrist with and without the support. A three-axial accelerometer (LSM303D, Microelectronics, Switzerland) was positioned with the $\mathrm{x}$-axis perpendicular to the sliding axis of the orthosis (Fig. 1) on the forearm mould support (Fig. 1). The measure of stability was based on the estimate of displacement resulting from double integration of the measured acceleration on the X-axis, as no significant oscillations were observed in the other directions. Collected signals were sampled at $50 \mathrm{~Hz}$ with the arm laid on a flat surface at $0^{\circ}$. Linear and nonlinear detrending methods were applied as described in [1] to the raw signals, for eliminating the influence of gravity and other low frequency components over the signal.

\subsection{Muscular Strength}

People suffering from PD experience reduced muscular strength. Hence, the relative muscular force for common daily tasks required by able-bodied persons is much lower than those of individuals with PD. Therefore, muscular strength (MS) was identified as a relevant ergonomic variable.

The Maximum Voluntary Muscular Contraction (MVC) of a healthy male was collected and analysed. The MVC was acquired during the resistive active movement of flexion and extension of the wrist over 12 seconds. This task was repeated three times with a two-minute rest interval to allow for adequate relaxation of the muscle fibre inbetween tests.

To acquire EMG signals, a customized system based on the Intan Tech's RHD2000 Evaluation System was employed. EMG was collected simultaneously from the flexor and extensor muscles of the subject's dominant forearm, with and without the orthosis. Disposable sensors (Meditrace, $35 \mathrm{~mm}$ ) were used for EMG detection.

EMG signals were filtered by a zero-phase high passband Butterworth digital filter $\left(6^{\text {th }}\right.$ order, $30 \mathrm{~Hz}$ cut-off frequency). The envelope of the signal was estimated by means of the root mean square value (RMS) and the mean of its main peaks (based on a predefined threshold) was employed as the main feature extracted from EMG signals. Signal analysis was performed by using customized programs developed in MatLab (Mathworks, USA).

Fig. 2 shows the test setup. Eight flexion-extension repetitions were carried out with the forearm-rest support in these angles: $0^{\circ}, 18^{\circ}, 45^{\circ}$ and $90^{\circ}$ relative to the horizontal line. This angular-variation study corresponds to the ergonomic variable of forearm positioning while wearing the orthosis. The EMG signal was expressed as a percentage of MVC. 

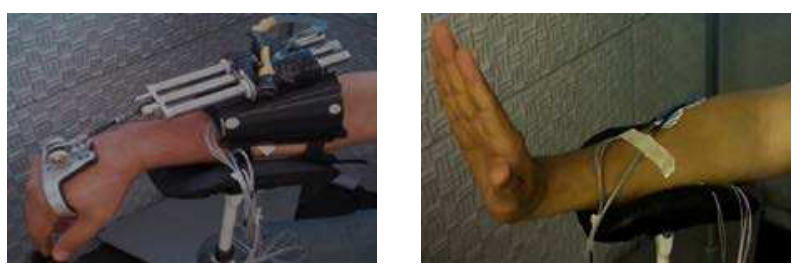

Fig. 2: The setup of the EMG sensors on the forearm, with and without the orthosis.

The importance of this ergonomic variable is that the desired design of the orthosis should require an MS that is quite low, often $10 \%$ in comparison with the user not wearing the orthosis. This investigation identified: (a) the percentage of the subject's MVC required to do a flexion or an extension for a given inclination angle of the forearm; and (b) the percentage of additional MVC introduced by using the orthosis.

\section{$4 \quad$ Results}

\subsection{Orthosis Stability}

Fig. 3 depicts a typical result obtained from a subject executing successive flexions and extensions, with and without the orthosis support.

The mean and standard error for the conditions with and without support were respectively $11.0 \pm 3.9 \mathrm{~mm}$ and $-7.0 \pm 17.7 \mathrm{~mm}$. The absolute amplitude was of $7.7 \mathrm{~mm}$ for the condition with support against $33.4 \mathrm{~mm}$ for the condition without the support. The relative difference between the absolute amplitudes was of $76.86 \%$, meaning that there was an improvement in the orthosis stability.

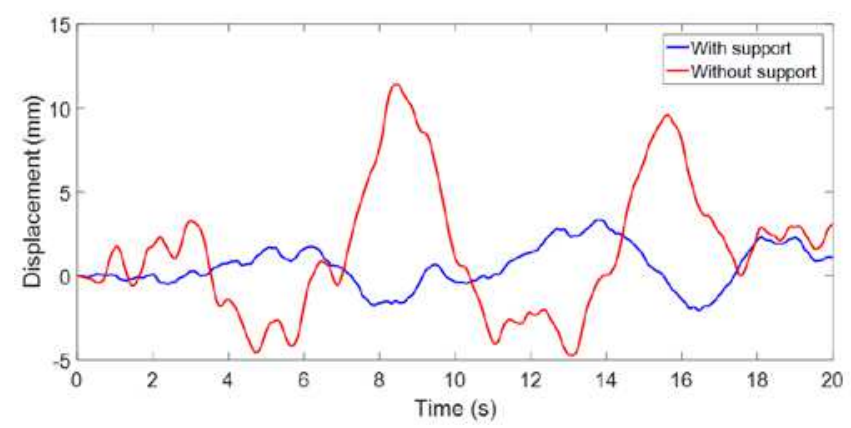

Fig. 3: Comparison of the displacement of the orthosis with and without the ergonomic support.

\subsection{Muscular Strength}

Fig. 4 shows the \%MVC for the different arm positions, with and without the orthosis. Fig. 5 shows the Ergonomic Index (EI) associated with the flexion and extension movements for the different angles, where the EI is defined according to Eq. 1, 
where $\% M V C_{\text {with orthosis }}$ and $\% M V C_{\text {without orthosis }}$ is the percentage MVC with and without the orthosis respectively.

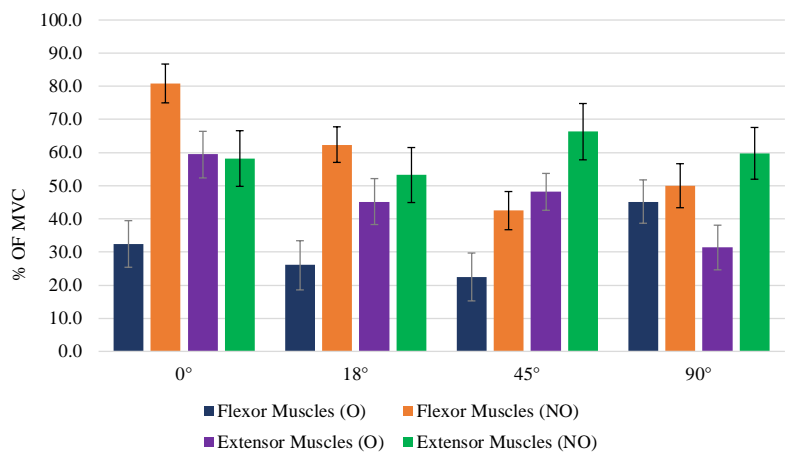

Fig. 4: Comparison of percentage MVC for flexor and extensor muscles. Note: $\mathrm{O}$ and NO refers to Orthosis and No Orthosis. The overall mean and standard deviation are presented.

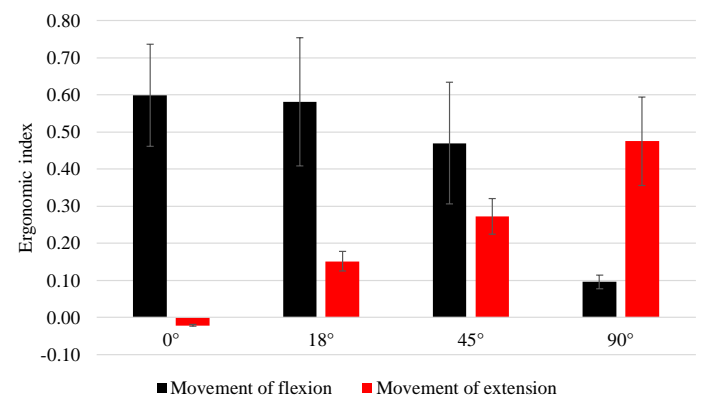

Fig. 5: Ergonomic Index from the flexion and extension movements at distinct angles. The overall mean and standard deviation are presented.

\section{$5 \quad$ Discussion and Conclusion}

In this study, it was presented an orthosis developed for rehabilitation of flexion and extension wrist activity following muscular stiffness resulting from PD. This communication has focused on the ergonomic assessment of the orthosis. An enhanced support structure has been designed and used with the orthosis and objectively shown to lead to a $76.86 \%$ stability improvement.

In Fig. 5 it is possible to verify an increase in the value of the EI for the extension movement as a function of the increase in the angle. This was expected because at $90^{\circ}$ the weight of the orthosis contributed to the movement. The same reasoning is true for 
the movement of flexion, in which there was a reduction of the EI as function as the decrease of the angle. This means that the EI, as it was defined, is not only capturing information about ergonomics but also on the way the muscles work. The results of this research have opened opportunities for further large-scale studies involving the use of the orthosis with healthy individuals and patients with PD.

\section{Acknowledgements}

The present work has the support of Brazilian government (CNPq, CAPES, FAPEMIGAPQ-00942-17) as well as the UK Royal Society International Exchange (IE170195). Adriano Pereira is a Fellow of CNPq, Brazil (306718/2014-6). A. O. Andrade is a Fellow of CNPq, Brazil (305223 / 2014-3).

\section{$7 \quad$ Conflicts of Interest}

The authors declare no conflict of interest.

\section{References}

1. Andrade AO, Ferreira LCV, Rabelo AG, Vieira MF, Campos AR, Goncalves BF, Pereira AA, Gonçalves BF, Pereira AA (2017) Pelvic movement variability of healthy and unilateral hip joint involvement individuals. Biomed Signal Process Control 32:1019. doi: 10.1016/j.bspc.2016.10.008

2. Christofoletti G, Cândido ER, Olmedo L, Miziara SRB, Beinotti F (2012) Efeito de uma intervenção cognitivo-motora sobre os sintomas depressivos de pacientes com doença de Parkinson. J Bras Psiquiatr 61:78-83. doi: 10.1590/S0047-20852012000200004

3. Dickson JM, Grünewald RA (2004) Somatic symptom progression in idiopathic Parkinson's disease. Parkinsonism Relat Disord 10:487-492. doi: 10.1016/j.parkreldis.2004.05.005

4. Fasoli SE, Krebs HI, Stein J, Frontera WR, Hogan N (2003) Effects of robotic therapy on motor impairment and recovery in chronic stroke. Arch Phys Med Rehabil 84:477482. doi: 10.1053/apmr.2003.50110

5. Rossi B, Siciliano G, Carboncini MC, Manca ML, Massetani R, Viacava P, Al. E (1996) Muscle modifications in Parkinson's disease: Myoelectric manifestations. Electroencephalogr Clin Neurophysiol - Electromyogr Mot Control 101:211-218

6. Sangha S, Elnady AM, Member S, Menon C (2016) A Compact Robotic Orthosis for Wrist Assistance. 1080-1085

7. Stefano M, Patrizia P, Mario A, Ferlini G, Rizzello R, Rosati G (2014) Robotic Upper Limb Rehabilitation after Acute Stroke by NeReBot: Evaluation of Treatment Costs. Biomed Res Int 2014:1-5. doi: 10.1155/2014/265634 